\title{
Evaluation agro-morphologique de sept écotypes de lentille de terre (Macrotyloma geocarpum) cultivés au Burkina Faso
}

\author{
Rasmata NANA $^{1 *}$, Saïdou BONKOUNGOU ${ }^{2}$, N'golo Diakalia COULIBALY $^{3}$, \\ Amos MININGOU², Bepio Hervé BAMA² et Hawa SOHORO ${ }^{2}$
}

\author{
${ }^{1}$ Laboratoire Biosciences ; Ecole Doctorale Sciences et Technologies, Université Ki-Zerbo, Burkina Faso 03 \\ BP 7021 Ouagadougou 03, Burkina Faso. \\ ${ }^{2}$ Institut de l'Environnement et de Recherches Agricoles (INERA), 04 BP 8645 Ouagadougou 04, \\ Burkina Faso. \\ ${ }^{3}$ Direction Générale de la Promotion de l'Economie Rurale (DGPER)/Ministère de l'Agriculture et des \\ Aménagements Hydro-agricoles, Burkina Faso. \\ *Auteur correspondant ; E-mail : nanaras2001@yahoo.fr; Tél: +226 70177933
}

\section{RESUME}

Macrotyloma geocarpum est une légumineuse dont sa culture est largement abandonnée au profit de celle d'autres légumineuses. Cette étude avait pour objectif d'identifier les écotypes performants à partir d'une caractérisation agromorphologique dans les conditions pluviales. Les résultats ont montré une diversité phénotypique entre les écotypes pour les caractères couleur du tégument, couleur des fleurs et du pétiole. L'analyse de variance a révélé une faible variabilité entre les caractères quantitatifs. La classification ascendante hiérarchique a mis en évidence 3 ensembles de diversité agro-morphologique. Le premier groupe constitué de 3 écotypes à graines noires, est caractérisé par un nombre important de feuilles trifoliolées à la floraison, un cycle du semis à la floraison intermédiaire et un poids de 100 bonnes graines élevé. Le second groupe compte un seul écotype à graines blanches, caractérisé par un cycle de floraison précoce, un nombre de feuilles trifoliolées faible et un poids de 100 bonnes graines faible. Le troisième groupe constitué de 3 écotypes à graines noires, est caractérisé par un cycle de floraison tardif, un nombre de feuilles trifoliolées moyen et un poids de 100 bonnes graines moyen. Ces résultats pourront contribuer à la création de variétés performantes répondant aux attentes des producteurs et consommateurs.

(C) 2019 International Formulae Group. All rights reserved

Mots clés: Culture négligée, génotypes, caractères, variabilité, climat.

\section{Agro-morphological evaluation of seven earth lens (Macrotyloma geocarpum) ecotypes cultivated in Burkina Faso}

\begin{abstract}
Macrotyloma geocarpum is a legume whose cultivation is largely abandoned in favor of that of other legumes. The objective of this study was to identify successful ecotypes based on agromorphological characterization in rainfed conditions. The results showed phenotypic diversity between the ecotypes for seed coat color, flower color and petiole color. Analysis of Variance revealed little variability between quantitative
\end{abstract}


traits. The hierarchical ascending classification has highlighted 3 sets of agro-morphological diversity. The first group consisting of 3 black-seeded ecotypes is characterized by a large number of trifoliate leaves at flowering, a cycle of sowing at intermediate flowering and a high weight of 100 good seeds. The second group has only one white-seeded ecotype characterized by an early flowering cycle, a low trifoliate leaf count and a low good seed weight of 100. The third group of 3 black-seeded ecotypes is characterized by a late flowering cycle, a medium number of trifoliate leaves and a medium weight of 100 good seeds. These results can contribute to the creation of high-performance varieties that meet the expectations of producers and consumers.

(C) 2019 International Formulae Group. All rights reserved

Keywords: Neglected crop, Genotypes, characters, variability, climate.

\section{INTRODUCTION}

L'espèce Macrotyloma geocarpum ([Harms] Maréchal \& Baudet) communément appelée lentille de terre est une espèce légumineuse annuelle cultivée pour ses graines comestibles. En effet, les graines de cette légumineuse sont caractérisées par une forte densité énergétique et nutritionnelle. Au-delà de la stricte couverture des besoins nutritionnels, les graines de légumineuses, notamment les légumes secs, présentent des atouts indéniables pour la santé (Rémond et Walrand, 2017). La production des légumineuses alimentaires, riches en protéines pourraient contribuer à l'amélioration de l'état nutritionnel de la population, en particulier celui des femmes enceintes et des enfants. La lentille de terre qui est une légumineuse très nutritive, rustique, constitue une alternative pour relever le défi de la malnutrition chez les ménages à revenu moyen au Burkina Faso. En effet, ses graines sont riches en glucides qui contribuent pour $73 \%$ à l'énergie de la graine de la lentille de terre (Badiel, 2013) et en protéines. Malheureusement, la lentille de terre fait partie de la catégorie des espèces dites "orphelines" en raison de la confidentialité de sa production (Cullis et Kunert, 2017). Malgré le rôle que pourrait jouer la lentille de terre dans la sécurité alimentaire des populations, son introduction dans le système de recherche nationale reste marginale. De plus, le rapport général sur la campagne agricole des quatre dernières années ne mentionne aucunement la production de la lentille de terre au Burkina Faso. Ainsi, sa culture est largement abandonnée au profit de celle d'autres légumineuses plus productives et plus rentables, comme le niébé, l'arachide, le soja et le pois de terre. En plus, des études récentes au Burkina Faso ont révélé sa méconnaissance par la jeune génération dans les zones où elle est encore produite (Coulibaly, 2018). Il n'existe pas à ce jour d'études sur les performances agronomiques de la lentille de terre au Burkina Faso; les rares études réalisées les caractéristiques nutritionnelles de la graine (Badiel, 2013). Dans un souci de production continue de la lentille de terre, la présente étude a eu pour objectif global de mesurer les paramètres qualitatifs et quantitatifs des écotypes de lentille de terre cultivés au Burkina Faso. De façon spécifique, il s'est agit de :

- déterminer les caractères morphologiques et agronomiques des écotypes collectés ;

- identifier les écotypes pouvant être exploitables dans les programmes de vulgarisation et de sélection.

\section{MATERIEL ET METHODES Matériel végétal}

Le matériel végétal est constitué de sept écotypes de lentille de terre. Ces écotypes ont été choisis sur la base de leur période de collecte et de leur faculté germinative à la suite d'un test de germination. Les écotypes ont été collectés dans quatre provinces de la partie Ouest du Burkina Faso (Carte 1). Leurs caractéristiques sont présentées dans le Tableau 1.

\section{Méthodes}

Dispositif expérimental et conduite de l'essai

L'essai a été réalisé dans des bidons de 20 litres préalablement découpés sur la longueur pour créer une profondeur de semis optimal au bon développement des plants. Les bidons ont été ensuite désinfectés à l'eau de Javel et de petits trous ont été perforés pour faciliter le drainage de l'eau. Chaque bidon a été rempli de terre stérilisée à la vapeur pendant 
2 heures. Dans quatre bacs en fer représentant les répétitions de l'essai, ont été disposés 7 bidons par bac. Chaque bidon constitue une parcelle élémentaire dans laquelle dix graines d'un écotype donné ont été semées. Le démariage a été effectué 14 jours après la levée (JAL) pour ne retenir que cinq plants présentant une bonne vigueur dans chaque parcelle élémentaire.

L'essai a été mise en place le 11 Juin 2018. Les semences ont été traitées avec le fongicide/insecticide Calthio $\mathrm{C}$, à la dose de 4 $\mathrm{g} / \mathrm{Kg}$ de semences avant les semis. Un apport de fertilisants par application d'engrais NPK 14-23-14 à la dose de $100 \mathrm{~kg} / \mathrm{ha}$ a été effectué au $14^{\text {ème JAL. }}$

L'expérimentation a été conduite suivant un dispositif en blocs de Fisher complètement randomisés à quatre répétitions. Les différentes caractéristiques du dispositif sont :

- une superficie de $1,8 \mathrm{~m}^{2}$ par répétition;

- une superficie de $864 \mathrm{~cm}^{2}$ par parcelle élémentaire (bidon de $36 \mathrm{~cm} \times 24 \mathrm{~cm}$ );

- une superficie totale de $13,6 \mathrm{~m} 2$ pour la parcelle;

- 7 parcelles élémentaires par répétition;

- les écartements entre les poquets ont été de 5 $\mathrm{cm} \times 5 \mathrm{~cm}$.

\section{Collecte des données}

La collecte des données a consisté à mesurer les caractères qualitatifs et quantitatifs sur cinq plantes de chaque parcelle élémentaire.

Les paramètres qualitatifs ont été déterminés par appréciation visuelle et ont concerné la forme des premières feuilles simples à $30 \mathrm{JAS}$; la forme des feuilles trifoliolées au stade pleine floraison; la pubescence du pétiole des feuilles trifoliolées et la couleur des fleurs.

Les mesures des paramètres quantitatifs ont porté sur les caractères suivants :

- le pourcentage de levée (\%L) : il a été évalué en pourcent du nombre de plants levés, rapportés au nombre de graines semées par parcelle élémentaire, après le comptage du nombre de plants levés chaque jour jusqu'à 14 JAS;
- la longueur du pétiole des feuilles simples à 14 JAS (LPFS 14JAS) : la longueur du pétiole a été mesurée sur les premières feuilles à l'aide d'une règle graduée au $14^{\text {ème }}$ JAS ;

- la longueur du pétiole de la $1^{\text {ère }}$ feuille trifoliolée à 14 JAS (LPFT 14JAS); à 30 JAS (LPFT 30 JAS) et la longueur des pétiolules en pleine floraison (LPeFT): la longueur du pétiole de la $1^{\text {ère }}$ feuille trifoliolée et celle des pétiolules ont été mesurées à l'aide d'une règle graduée respectivement au $14^{\text {ème }}$; au $30^{\text {ème }} \mathrm{JAS}$ et en pleine floraison;

- le nombre total de feuilles trifoliolées à 14 JAS (NFT 14 JAS) et à 30 JAS (NFT 30 JAS): il a été évalué par comptage manuel du nombre total de feuilles trifoliolées au $14^{\text {ème }}$ et au $30^{\text {ème }} \mathrm{JAS}$;

- la date d'apparition de la première fleur (DAPF) : elle a été déterminée par observations quotidiennes des plantes afin de noter la première fleur apparue sur une plante de la parcelle élémentaire et ensuite l'évaluer en nombre de JAS ;

- la date de $50 \%$ floraison $(50 \% \mathrm{~F})$ : elle a été évaluée par comptage du nombre de jours écoulés entre la date de semis et le jour où $50 \%$ des plantes de la parcelle portaient au moins une fleur;

- la hauteur en pleine floraison (HPPF) : elle a été mesurée de la base de la tige principale jusqu'au bourgeon terminal pendant la pleine floraison :

- le poids de 100 bonnes graines (P100G) : le poids de cent bonnes graines a été déterminé par écotype à l'aide d'une balance de précision $0,01 \mathrm{~g}$.

\section{Analyses statistiques}

Les données collectées ont été analysées avec le logiciel XLSTAT version 2018 20.5.52064.0. L'analyse de variance (ANOVA) a été réalisée pour l'ensemble des caractères quantitatifs, la comparaison des moyennes a été faite selon le test de Tukey au seuil de 5\%. L'analyse en composantes principales (ACP) a été faite pour extraire les variables quantitatives les plus représentatives. Ces variables ont servi à la classification ascendante hiérarchique (CAH). 
Tableau 1: Caractéristiques des écotypes de lentille de terre.

\begin{tabular}{|c|c|c|c|c|}
\hline $\begin{array}{l}\text { Noms codés } \\
\text { des écotypes }\end{array}$ & $\begin{array}{l}\text { Couleur du } \\
\text { tégument de la } \\
\text { graine (photo 1) }\end{array}$ & $\begin{array}{l}\text { Nom de la } \\
\text { localité de } \\
\text { collecte } \\
\end{array}$ & $\begin{array}{l}\text { Province de } \\
\text { la localité de } \\
\text { collecte }\end{array}$ & $\begin{array}{l}\text { Région de la localité de } \\
\text { collecte }\end{array}$ \\
\hline Dou & Noire & Doufiguisso & Houet & Hauts Bassins \\
\hline Oky1 & Noire & Ouarkoye & Mouhoun & Boucle du Mouhoun \\
\hline $\operatorname{Sin} 1$ & Noire & Sindou & Léraba & Cascades \\
\hline Gna & Noire & Gnassogoni & Léraba & Cascades \\
\hline Oky2 & Noire & Ouarkoye & Mouhoun & Boucle du Mouhoun \\
\hline Uo & Noire & Dédougou & Mouhoun & Boucle du Mouhoun \\
\hline Oro & Blanche & Orodara & Kénédougou & Hauts Bassins \\
\hline
\end{tabular}

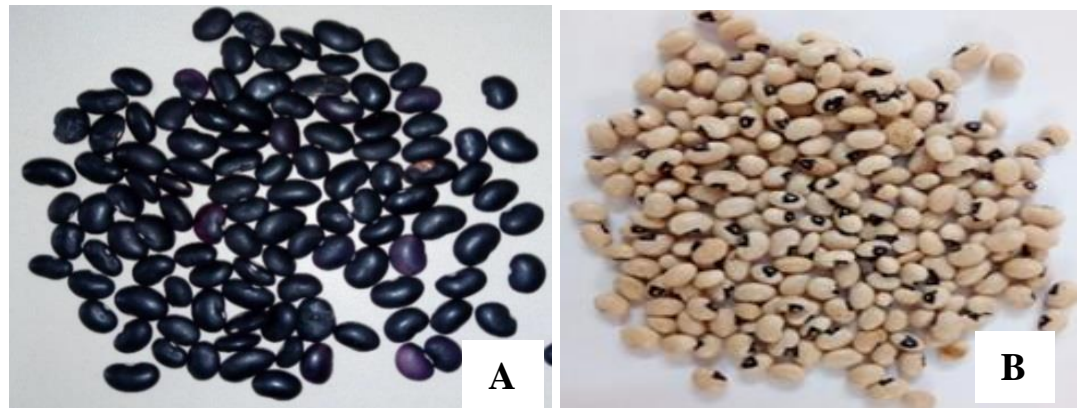

Photo 1 : Aspects de graines des différents écotypes de lentille de terre collectés : (A) graines noires au hile blanc; (B) graines blanches au hile blanc moucheté de noir.

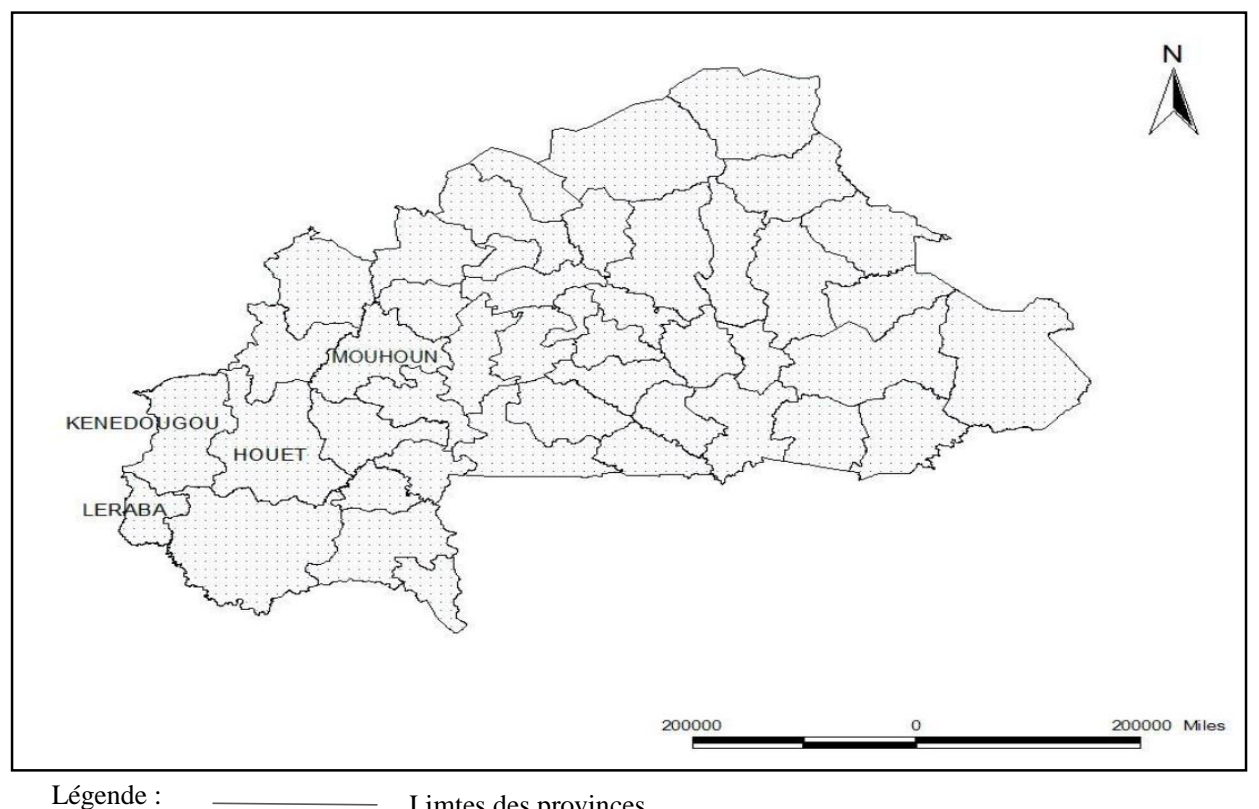

Carte 1: Localisation des provinces de collecphotote des écotypes de lentille de terre. 


\section{RESULTATS}

\section{Paramètres qualitatifs}

Formes des feuilles et pubescences des pétioles

Les observations visuelles portées sur les feuilles, ont permis de noter une absence de variation de la forme des premières feuilles entre les écotypes. En effet, les premières feuilles de tous les écotypes étudiés sont de forme lancéolée, simples et opposées. L'apparition des deux feuilles simples primordiales a eu lieu entre 6 à 10 jours après semis (JAS). En outre, les feuilles secondaires qui sont trifoliolées avaient une foliole terminale et les folioles latérales étaient toutes, de forme ovale. Ces caractéristiques ont été communes à tous les écotypes étudiés.

Quant à la pubescence des pétioles, il est ressorti des observations que les pétioles des feuilles trifoliolées des écotypes à graines noires (Oky1, Oky2, Sin1, Gna, Dou, Uo/Ded) sont de pilosité grise alors que celles de l'écotype à graines blanches (Oro) sont de couleur verte (photo 2).

\section{Couleurs des fleurs}

L'observation des fleurs a révélé une variation de couleurs et cette variation était fonction de la couleur des graines. Ainsi, les fleurs des écotypes à graines noires (Oky1, Oky2, Sin1, Gna, Dou, Uo/Ded) ont présenté des fleurs de couleur violette et l'écotype à graine blanche (Oro) avait des fleurs de couleur blanche (photo 3 ).

\section{Paramètres quantitatifs \\ Pourcentage de levée}

Il ressort des résultats une variabilité du taux de levée des différents écotypes avec un pourcentage moyen de levée faible chez l'écotype Oro (35\%) alors qu'un pourcentage de levée de $90 \%$ a été notée pour les écotypes Dou et Uo/Ded (Figure 1). L'analyse de variance a montré une différence hautement significative $(\mathrm{P}=0,001)$ entre l'écotype à graines blanches et les écotypes à graines noires pour le taux de levée (Tableau 2).

\section{Longueur des pétioles et des pétiolules}

Les résultats ont montré une grande variabilité entre les écotypes pour les caractères longueur des pétioles et celle des pétiolules. L'analyse de variance a révélé une différence très hautement significative entre les écotypes pour les caractères longueur du pétiole de la première feuille trifoliolée à 14 JAS et à 30 JAS et pour le caractère longueur du pétiolule en pleine floraison (Tableau 2). En effet, l'écotype Oro a présenté des pétioles plus longs avec en moyenne $7,9 \mathrm{~cm}$ et $18,4 \mathrm{~cm}$ respectivement à $14 \mathrm{JAS}$ et à $30 \mathrm{JAS}$ (Figure 2). Les plus longs pétiolules avec en moyenne $2,1 \mathrm{~cm}$ ont été mesuré chez les écotypes Dou et Oky1. La longueur du pétiole des feuilles simples à 14 JAS a été significativement différente entre les écotypes $(\mathrm{P}=0,009)$.

\section{Nombre de feuilles trifoliolées}

Les résultats de l'analyse de variance (Tableau 2) ont fait ressorti une différence significative entre les écotypes pour les caractères nombre de feuilles trifoliolées à 14 JAS $(\mathrm{P}=0,044)$ et nombre de feuilles trifoliolées à 30 JAS $(P=0,029)$. Pour ces deux caractères, l'écotype Oro a montré un nombre moyen de feuilles trifoliolées plus important au $14^{\text {ème }} \mathrm{JAS}$ et $30^{\text {ème }} \mathrm{JAS}$ soit respectivement 3 et 9 feuilles (Figure 3).

\section{Cycle de floraison}

L'analyse de variances (Tableau 2) a montré une différence hautement significative entre les écotypes étudiés pour les caractères date d'apparition de la $1^{\text {ère }}$ fleur (DAPF) et date de 50\% floraison. Cela témoigne de la diversité entre les écotypes pour ces deux caractères. L'écotype Oro a été relativement précoce par rapport aux autres écotypes avec environ 50 jours après semis pour l'apparition de la $1^{\text {ère }}$ fleur et 53 jours après semis pour le caractère $50 \%$ floraison (Figure 4). Cependant, le cycle semi-floraison le plus tardif a été observé chez les écotypes Oky 1 et Oky 2 dont la moitié floraison a été observée au $65^{\text {ème }}$ jour après semis, soit 15 jours d'écart avec la date 50ù floraison de l'écotype précoce (Oro).

\section{Hauteur des plantes en pleine floraison}

La hauteur des plantes en pleine floraison a varié de 39 à $43,90 \mathrm{~cm}$ entre les écotypes. L'écotype Sin1 a exprimé la faible hauteur avec en moyenne $39 \mathrm{~cm}$, les plants les plus hauts étant relevés au niveau de l'écotype Uo (Figure 5). Cependant, l'analyse de 
variance n'a révélé aucune différence significative $(P=0,21)$ entre les écotypes pour la variable hauteur des plantes en pleine floraison (Tableau 2).

\section{Poids de 100 bonnes graines}

Le poids de cent bonnes graines avec un poids moyen de 10,84 g pour l'ensemble des écotypes étudiés, a été variable en fonction des écotypes. En effet, l'écotype Gna a enregistré un poids moyen de 100 bonnes graines plus faible $(7 \mathrm{~g})$ alors que le poids moyen de 100 bonnes le plus élevé $(14,4 \mathrm{~g})$ a été noté chez l'écotype Sin1 (Figure 6). L'analyse de variance a révélé une différence très hautement significative $(\mathrm{P}<0,0001)$ entre les écotypes pour le caractère poids de cent bonnes graines (Tableau 2).

\section{Structuration de la diversité des écotypes de Macrotyloma geocarpum collectés}

La classification ascendante hiérarchique (Figure 7) a permis une répartition des sept écotypes en 3 classes. Ces classes sont constituées respectivement de 3,1 et 3 écotypes. En effet, la classe 1 est constituée des écotypes Oky1, Uo et Sin1. Cette classe regroupe les écotypes caractérisés par un début de floraison intermédiaire, un poids de 100 graines plus élevé et un nombre relativement élevé des feuilles trifoliolées en pleine floraison. La classe 2, se compose de l'écotype Oro qui est caractérisé par la couleur blanche de ses graines, un cycle de floraison précoce, un poids de 100 graines faible et un nombre assez faible des feuilles trifoliolées en pleine floraison. La classe 3 regroupe les écotypes Oky2, Dou, Gna, qui sont des écotypes caractérisés par un cycle de floraison tardif, avec un poids de 100 graines assez moyen et un nombre moyen de feuilles trifoliolées en pleine floraison.

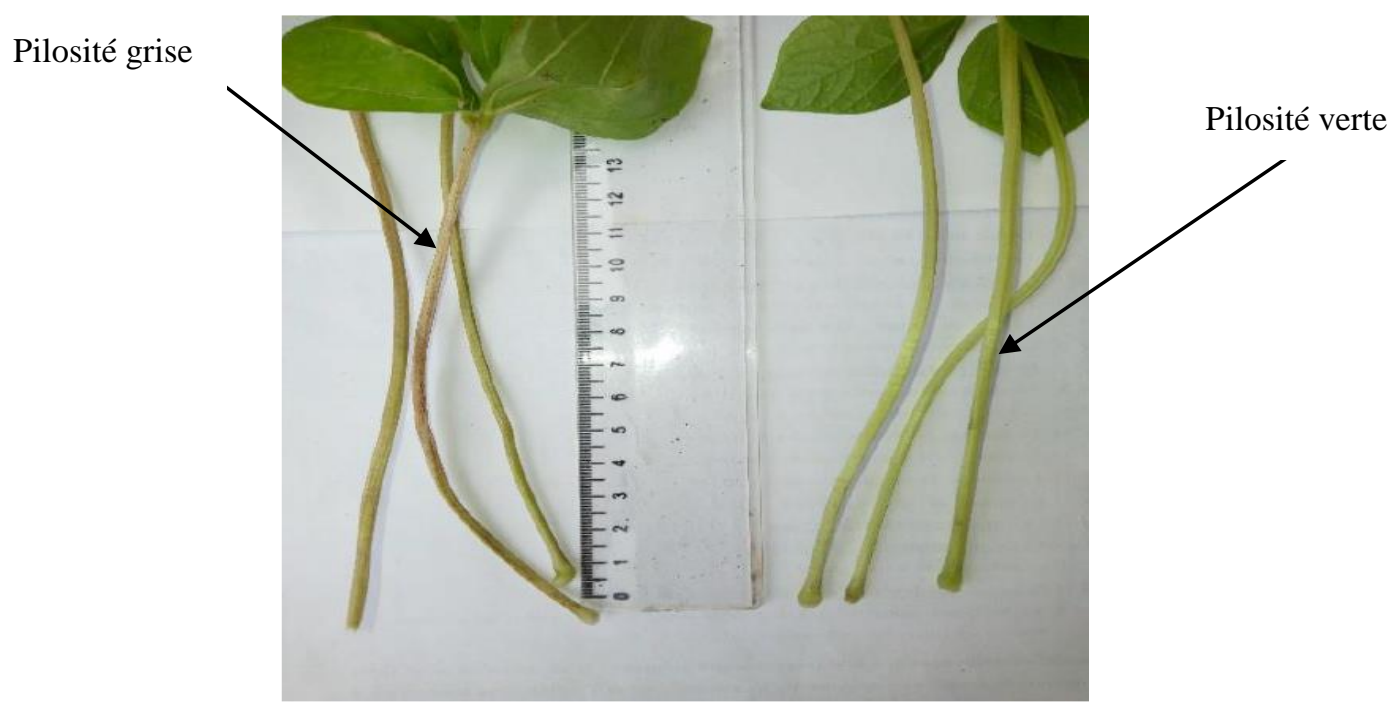

Photo 2 : Pilosité des feuilles trifoliolées de la lentille. 

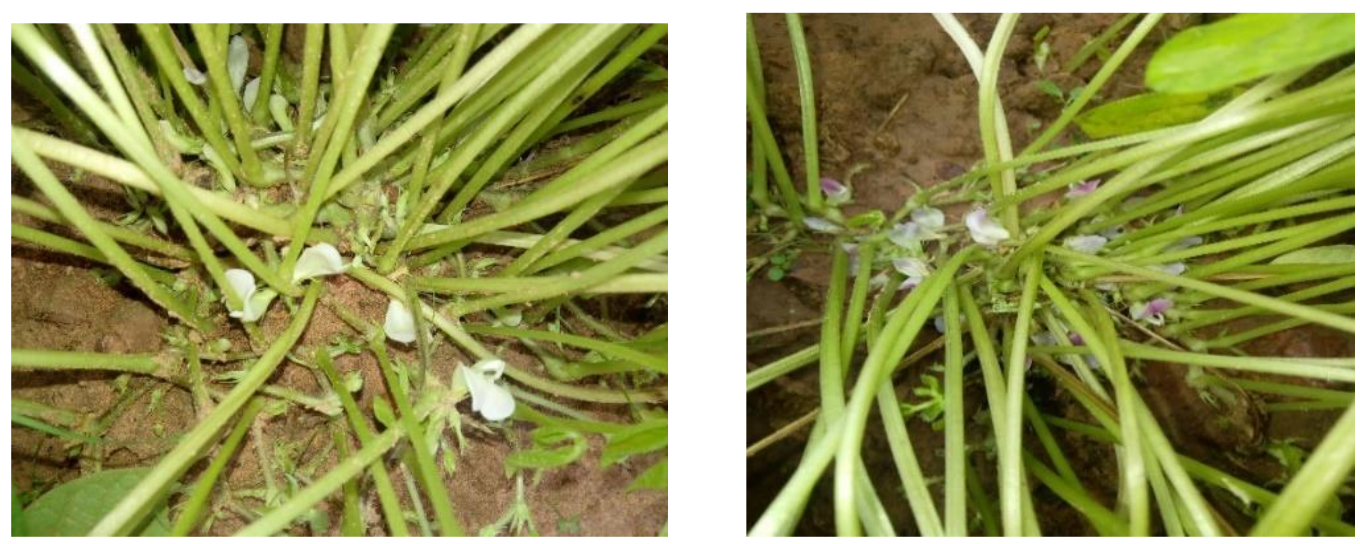

Photo 3: Couleurs des fleurs de lentilles de terre (fleurs blanches à gauche et fleurs violettes à droite)

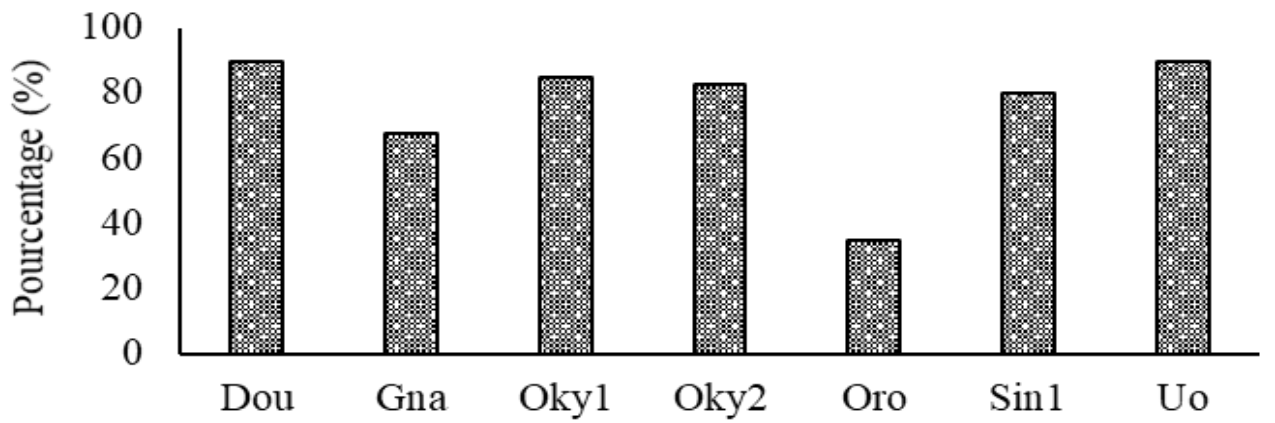

\section{Ecotypes}

Figure 1 : Pourcentage de levée des écotypes de lentille de terre étudiés.

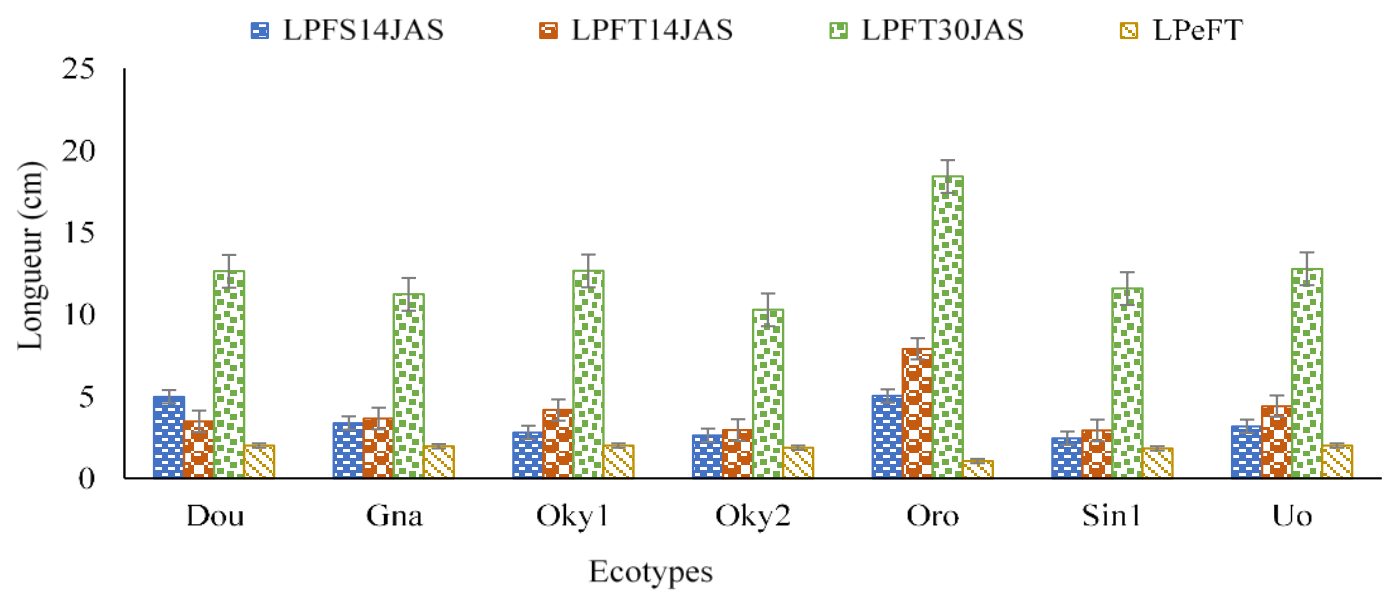

Légende : LPFS14JAS = Longueur du pétiole des feuilles simples à 14 JAS ; LPFT14JAS = Longueur du pétiole de la 1 ìre feuille trifoliolée à 14 JAS ; LPFT30JAS : Longueur du pétiole de la $1^{\text {ère }}$ feuille trifoliolée à $30 \mathrm{JAS}$; LPeFT = Longueur des pétiolules de la $1^{\text {ère }}$ feuille trifoliolée en pleine floraison.

Figure 2 : variation de la longueur des pétioles et des pétiolules. 
Tableau 2 : Analyse de variance des caractères étudiés.

\begin{tabular}{|c|c|c|c|c|c|c|c|c|c|c|c|}
\hline \multirow[b]{2}{*}{$\begin{array}{l}\text { Variétés et } \\
\text { sources de } \\
\text { variation }\end{array}$} & \multirow[b]{2}{*}{$\% \mathrm{~L}$} & \multirow[b]{2}{*}{$\begin{array}{l}\text { LPFS 14JAS } \\
(\mathrm{cm})\end{array}$} & \multicolumn{9}{|c|}{ Variables } \\
\hline & & & $\begin{array}{c}\text { LPFT 14JAS } \\
(\mathrm{cm})\end{array}$ & $\begin{array}{l}\text { LPFT } \\
\text { 30JAS }\end{array}$ & $\begin{array}{c}\text { NFT } \\
\text { 14JAS }\end{array}$ & NFT 30JAS & $\begin{array}{c}\text { LPeFT } \\
(\mathbf{c m})\end{array}$ & DAPF & $50 \% \mathrm{~F}$ & $\begin{array}{c}\text { HPPF } \\
(\mathbf{c m})\end{array}$ & $\begin{array}{c}\text { P100Gr } \\
\text { (g) }\end{array}$ \\
\hline Uo & $90,00 \mathrm{a}$ & $3,18 \mathrm{ab}$ & $4,40 \mathrm{~b}$ & $12,78 \mathrm{~b}$ & $2,00 \mathrm{ab}$ & $8,25 \mathrm{ab}$ & $1,99 \mathrm{a}$ & $59,25 \mathrm{a}$ & $63,25 \mathrm{a}$ & $43,90 \mathrm{a}$ & $13,50 \mathrm{a}$ \\
\hline Oky1 & $85,00 \mathrm{a}$ & $2,80 \mathrm{ab}$ & $4,18 b$ & $12,65 \mathrm{~b}$ & $1,75 \mathrm{ab}$ & $8,50 \mathrm{ab}$ & $2,01 \mathrm{a}$ & $59,00 \mathrm{a}$ & $65,25 \mathrm{a}$ & $42,65 \mathrm{a}$ & $12,50 \mathrm{a}$ \\
\hline Oro & $35,00 \mathrm{~b}$ & $5,03 \mathrm{a}$ & $7,90 \mathrm{a}$ & $18,40 \mathrm{a}$ & $2,50 \mathrm{a}$ & $9,25 \mathrm{a}$ & $1,05 \mathrm{~b}$ & $50,25 \mathrm{~b}$ & $52,75 \mathrm{~b}$ & $40,50 \mathrm{a}$ & $9,70 \mathrm{a}$ \\
\hline Dou & $90,00 \mathrm{a}$ & $4,98 \mathrm{a}$ & $3,48 \mathrm{~b}$ & $12,63 \mathrm{~b}$ & $1,50 \mathrm{ab}$ & $7,50 \mathrm{ab}$ & $2,01 \mathrm{a}$ & $58,00 \mathrm{a}$ & $62,75 \mathrm{a}$ & $43,60 \mathrm{a}$ & $9,00 \mathrm{ab}$ \\
\hline Oky2 & $82,50 \mathrm{a}$ & $2,63 a b$ & $2,95 \mathrm{~b}$ & $10,28 \mathrm{~b}$ & $1,50 a b$ & $6,50 \mathrm{ab}$ & $1,88 \mathrm{a}$ & $62,75 \mathrm{a}$ & $65,00 \mathrm{a}$ & $41,90 \mathrm{a}$ & $9,80 \mathrm{a}$ \\
\hline Gna & $\begin{array}{c}67,50 \\
\mathrm{ab}\end{array}$ & $3,38 \mathrm{ab}$ & $3,65 \mathrm{~b}$ & $11,23 \mathrm{~b}$ & $1,75 \mathrm{ab}$ & $6,25 \mathrm{ab}$ & $1,96 \mathrm{a}$ & 59,75 a & $62,50 \mathrm{a}$ & $41,15 \mathrm{a}$ & $7,00 \mathrm{ab}$ \\
\hline Sin1 & $80,00 \mathrm{a}$ & $2,45 \mathrm{~b}$ & $2,93 \mathrm{~b}$ & $11,58 \mathrm{~b}$ & $1,25 \mathrm{~b}$ & $4,75 \mathrm{~b}$ & $1,82 \mathrm{a}$ & $59,25 \mathrm{a}$ & $63,75 \mathrm{a}$ & $39,00 \mathrm{a}$ & $14,40 \mathrm{a}$ \\
\hline Moyenne & 75,71 & 3,49 & 4,21 & 12,79 & 1,75 & 7,29 & 1,819 & 58,75 & 62,18 & 41,81 & 10,84 \\
\hline $\operatorname{CV}(\%)$ & 30,17 & 40,15 & 45,26 & 22,81 & 33,45 & 29,62 & 18,698 & 7,49 & 7,39 & 7,11 & 23,282 \\
\hline $\mathbf{R}^{2}$ & 0,649 & 0,527 & 0,724 & 0,727 & 0,432 & 0,459 & 0,922 & 0,744 & 0,774 & 0,307 & 0,009 \\
\hline $\mathbf{P}$ & 0,001 & 0,009 & $<0,0001$ & $<0,0001$ & 0,044 & 0,029 & $<0,0001$ & $<0,0001$ & $<0,0001$ & 0,210 & $<0,0001$ \\
\hline $\begin{array}{l}\text { Significati } \\
\text { on }\end{array}$ & $* * *$ & $* * *$ & $* * * *$ & $* * * *$ & $* * *$ & $* * *$ & $* * * *$ & $* * * *$ & $* * * *$ & ns & $* * * *$ \\
\hline
\end{tabular}

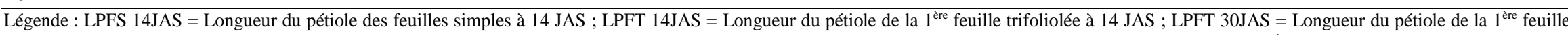

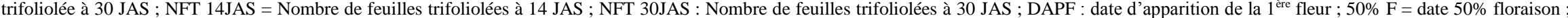

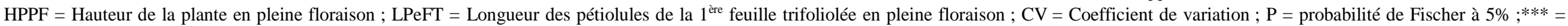
hautement significatif $; * * * *=$ très hautement significatif $; \mathrm{ns}=$ non significatif.

Les valeurs d'une même colonne, suivies de la même lettre ne sont pas significativement différentes de la point de vue statistique. 


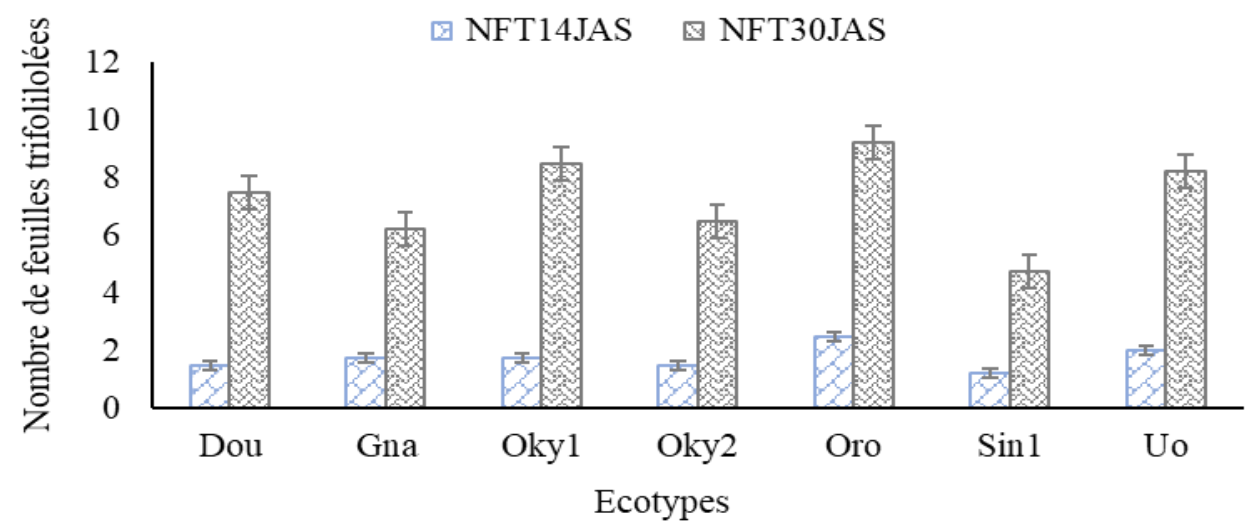

Légende : NFT14JAS = Nombre de feuilles trifoliolées à 14 JAS ; NFT 30JAS = Nombre de feuilles trifoliolées à 30 JAS.

Figure 3 : Feuilles trifoliolées des écotypes aux $14^{\text {ème }}$ et $30^{\text {ème }} \mathrm{JAS}$.

DAPF $50 \% \mathrm{~F}$

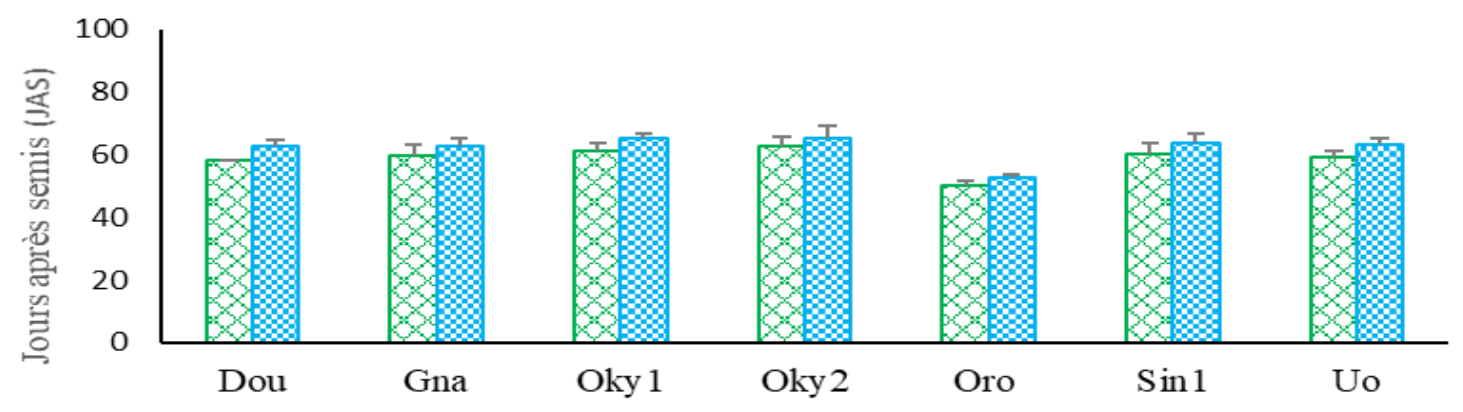

Ecotypes

Légende : DAPF = date d'apparition de la $1^{\text {ère }}$ fleur $; 50 \% \mathrm{~F}=$ date $50 \%$ floraison.

Figure 4 : Dates de floraison des écotypes en JAS.

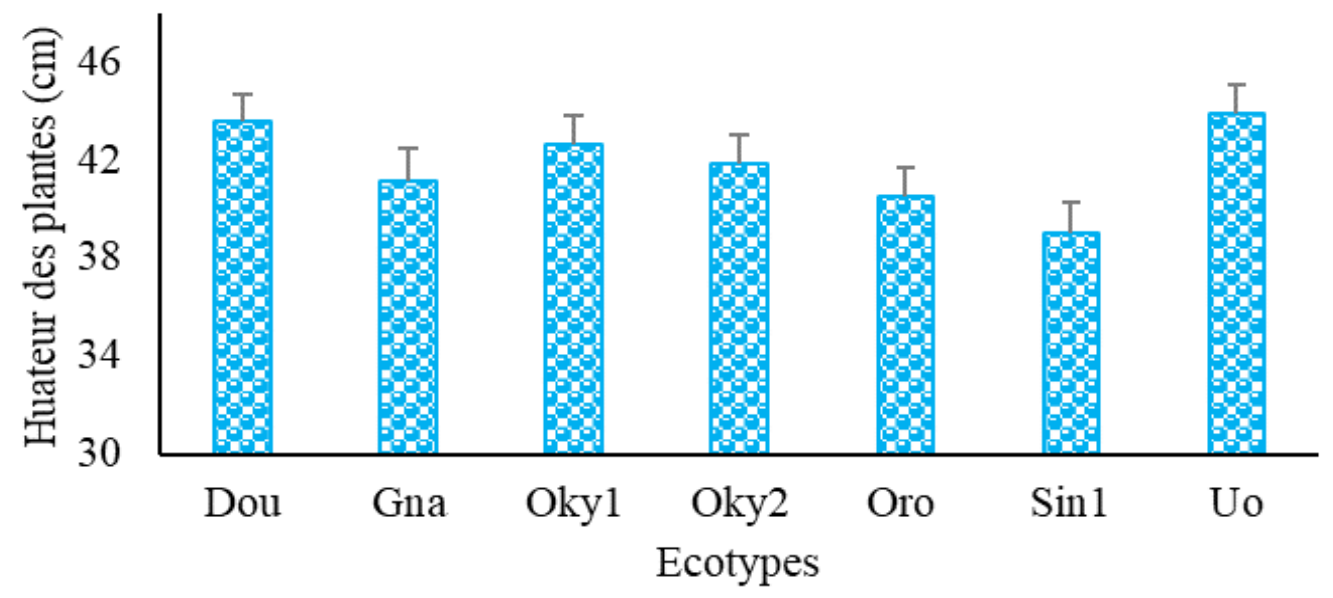

Figure 5 : Hauteur moyenne des plantes en pleine floraison des écotypes de lentille de terre. 


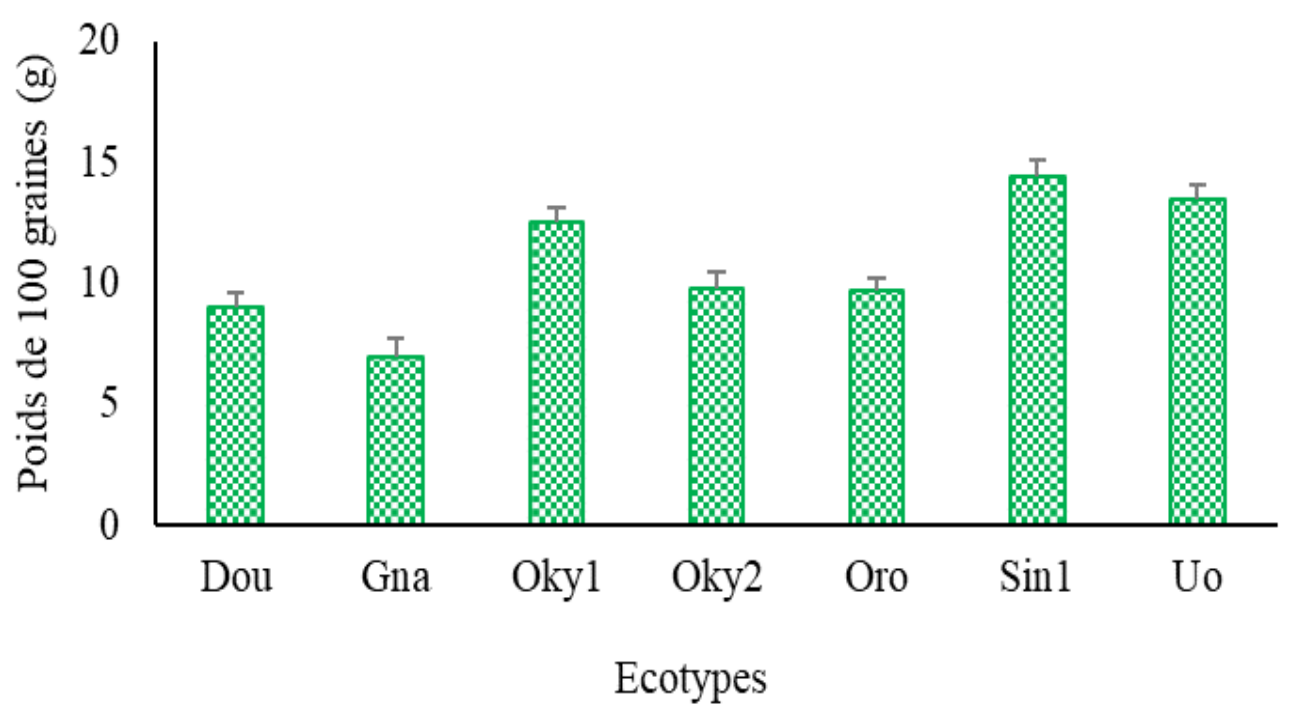

Figure 6 : Poids de 100 bonnes graines des écotypes.

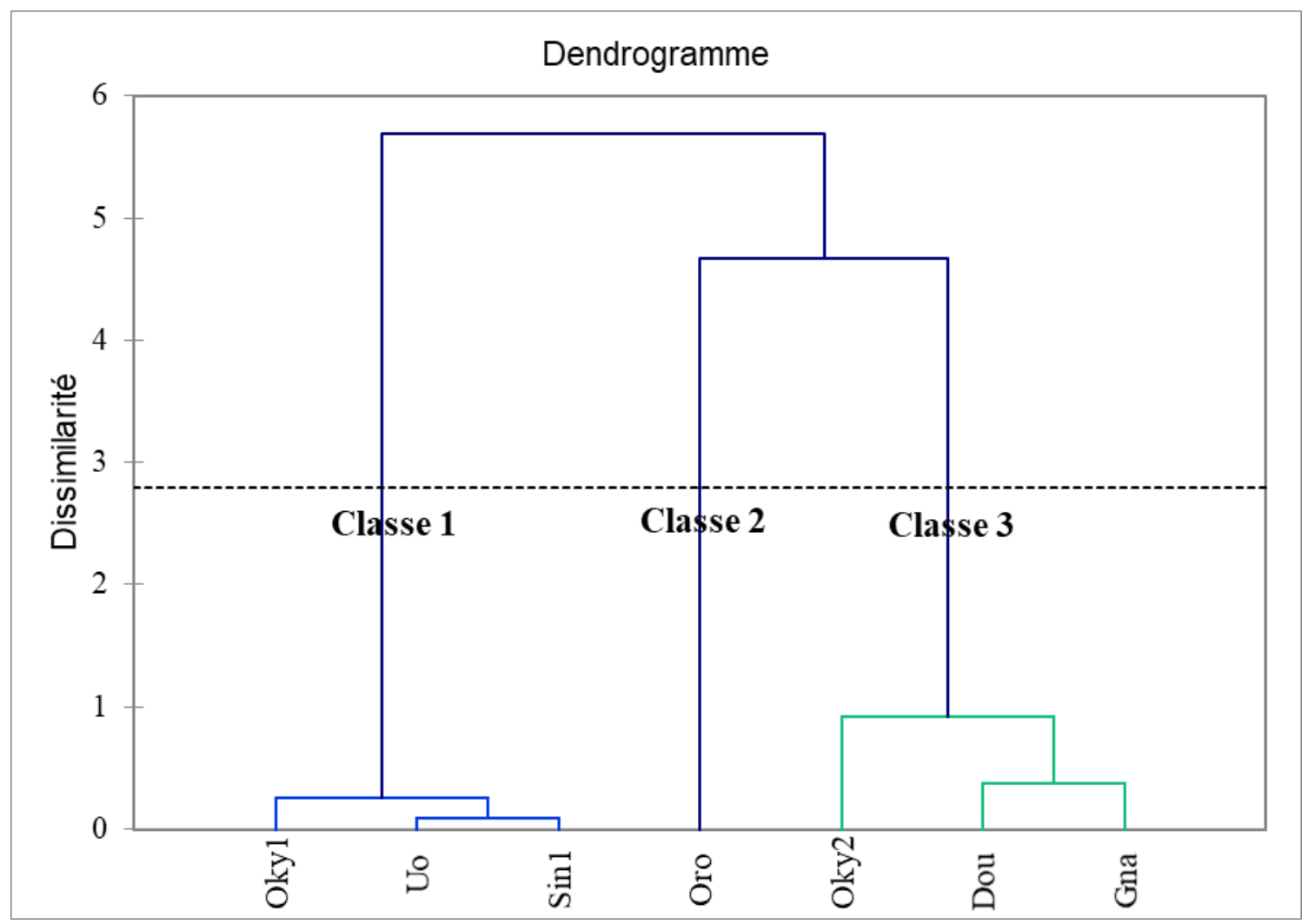

Figure 7 : Dendrogramme issu de la classification ascendante hiérarchique. 


\section{DISCUSSION}

Les résultats sur l'observation des caractères qualitatifs ont permis de distinguer deux types d'écotypes. Le premier type constitué de l'écotype Oro, aux fleurs blanches et aux pétioles de pilosité verte se distingue nettement du deuxième type constitué des écotypes Oky1, Oky2, Sin1, Gna, Dou, Uo qui sont caractérisés par des fleurs violettes et des pétioles de pilosité grise. Les couleurs observées sur les fleurs sont en conformité avec les résultats de Duke et al. (1977) qui ont affirmé que les lentilles de terre à graines blanches produisent des fleurs blanches tandis que celles à graines rouges, noires ou pigmentées produisent des fleurs de couleur pourpre. Toutefois, les écotypes ont été assez homogènes pour les caractères qualitatifs.

L'analyse de variance a révélé une différence très hautement significative pour les caractères longueur du pétiole de la première feuille trifoliolée à 14 JAS et à 30 JAS, la longueur du pétiolule en pleine floraison, la date d'apparition de la 1 ère fleur, la date de $50 \%$ floraison, le poids de cent bonnes graines. Ces résultats montrent que ces paramètres sont très déterminants pour expliquer la variabilité phénotypique de Macrotyloma geocarpum et pourrait exprimer des spécificités génotypiques des différents écotypes. Selon Sévérin et al. (2011), la variabilité phénotypique observée chez Vigna subterranea pourrait résulter de l'expression d'une forte hétérogénéité génotypique mais aussi de l'influence des facteurs environnementaux.

Les résultats sur la floraison ont montré une précocité de la floraison de l'écotype Oro pour les caractères DAPF et $50 \%$ floraison soit 50 JAS pour la date de la première floraison et $53 \mathrm{JAS}$ pour la date de $50 \%$ floraison contre une moyenne supérieure à $60 \mathrm{JAS}$ pour la date de la première floraison et $50 \%$ floraison pour les autres écotypes. Ces résultats traduiraient un développement plus rapide de l'écotype Oro par rapport aux autres écotypes. La différence entre les parties végétatives des plantes peut s'expliquer également par l'influence des facteurs environnementaux. En effet, la variabilité de croissance entre des morphotypes de voandzou, plante de la même famille que la lentille de terre (Touré et al., 2013) pourrait être liée à l'efficience d'interception du rayonnement solaire et surtout à l'efficience biologique d'interception de ce rayonnement. Dans une telle situation, la maîtrise de la période de semis est un facteur déterminant pour garantir une bonne productivité de la lentille de terre. Les différences non significatives enregistrées pour certains caractères montrent une certaine homogénéité des écotypes pour ces variables. L'analyse de variance a révélé des effets significatifs des caractères longueur du pétiole, nombre de feuilles trifoliolées, cycle de floraison et poids de 100 graines sur les écotypes étudiés. Ces résultats dénotent d'une certaine variabilité inter-écotypes pour ces caractères. Ainsi, Gahlmi (2017) a trouvé que la variabilité entre des écotypes de niébé était plus grande pour les traits quantitatifs que pour ceux qualitatifs.

$\mathrm{La}$ classification ascendante hiérarchique $(\mathrm{CAH})$ a permis de regrouper les écotypes étudiés en trois classes dont l'une de leurs caractéristiques est la différence du cycle de développement. Toutefois, Brink et Belay (2006) ont rapporté que la durée du cycle cultural de la lentille de terre varie de 90 à 180 jours. Par ailleurs, chez la lentille de terre, les fleurs se formant à l'aisselle des feuilles, un grand nombre de feuilles trifoliolées pourraient influencer la formation d'un nombre important de fleurs et donc favoriser la formation d'une quantité importante de gousses. Les résultats du CAH ont mis en évidence 3 classes de diversité, caractérisées par des dissemblances morphologiques et phénotypiques qui pourraient être liées aux facteurs physiques de l'environnement non identiques à ceux de la provenance de chaque écotype. En effet, Bonny et Dje (2011) ont souligné que les 
dissemblances et phénologiques entre des groupes phénotypiques de Vigna subterranea seraient dû à la maintenance des accessions sous des processus évolutifs très différents de leur agrosystème respectif. $\mathrm{Au}$ regard des résultats de la $\mathrm{CAH}$, la classe 1 se présente comme la classe des écotypes performants car ces écotypes pourraient engendrer un rendement élevé, qui est l'objectif recherché par les producteurs. Les écotypes de la classe 1 constitueraient alors une source potentielle pour les programmes de sélection et d'amélioration. Cependant, les écotypes de la classe 3 devraient être semés juste au début de la saison pluvieuse au regard de leur cycle tardif. L'écotype Oro qui a un cycle de floraison précoce pourrait être exploité dans les zones à faibles pluviométrie du Burkina Faso.

\section{Conclusion}

Cette étude sur la caractérisation morphologique et agronomique des écotypes de lentille de terre cultivée au Burkina Faso avait pour objectif d'identifier les écotypes performants. Ainsi, l'étude a révélé une variabilité entre les écotypes pour les caractères étudiés. Cette variabilité a été structurée en 3 groupes de diversité caractérisée par le nombre de feuilles trifoliolées, la durée du semis à la floraison et le poids de 100 bonnes graines. Les analyses de variance laissent apparaître que les écotypes Sin1, Oky1 et Uo/Ded à graines noires présentent de bonnes caractéristiques agronomiques. La variabilité exprimée par les écotypes de lentille de terre constitue un indicateur qui pourrait être exploitée par les programmes d'amélioration variétale de cette espèce.

\section{CONFLITS D'INTERETS}

Les auteurs déclarent qu'il n'y a pas de conflit d'intérêts en ce qui concerne la publication de cet article.

\section{CONTRIBUTIONS DES AUTEURS}

$\mathrm{RN}$ et SB: Elaboration des fiches de collectes des données, supervision de l'expérimentation et la collecte des données, réalisation des analyses statistiques et la rédaction du manuscrit; NDC: ProspectionCollecte des écotypes dans les différentes localités, collecte des données d'expérimentation, saisie des données, analyses statistiques et participation à la rédaction du manuscrit; $\mathrm{AM}$ : Participation à la rédaction du manuscrit; $\mathrm{BHB}$ et $\mathrm{HS}$ : Prospection-Collecte des écotypes dans les différentes localités et accompagnement dans la collecte des données d'expérimentation.

\section{REMMERCIEMENTS}

Nos sincères remerciements vont à l'endroit de l'équipe du programme oléoprotéagineux DRREA Ouest/Station de Recherches Agricoles de Farako-Bâ et au Laboratoire Biosciences de l'Université Joseph KI-ZERBO.

\section{REFERENCES}

Badiel B. 2013. Dosage de différents constituants biochimiques et mise en évidence des caractéristiques nutritionnelles des graines de lentille de terre (Macrotyloma geocarpum [Harms] Marechal et Baudet). Mémoire DEA, Université de Ouagadougou, Ouagadougou, p. 53.

Bonny SB, Djé Y. 2011. Variabilité morphologique et agronomique de variétés traditionnelles de Voandzou (Vigna subterranea) (L.) Verdc. (Fabaceae) de Côte d'Ivoire. Journal of Applied Biosciences, 41: 2820-2835. DOI : https://dx.doi.org/10.4314/jab.v138i1.1

Brink M, Belay G. 2006. Ressources végétales de l'Afrique tropicale. Céréales et légumes secs. Fondation prota, wage ningen, pays Bas/Backhuys publishers, 
Leiden, pays Bas/CTA, Wageningen, Pays Bas, p. 328.

Cullis C, Kunert KJ. 2017. Unlocking the potential of orphan legumes. Journal Experimental Botany, 68(8) : 1895-1903. DOI : $10.1093 /$ jxb/erw437.

Coulibaly ND. 2018. Caractérisation agromorphologique et comportement vis-àvis des maladies fongiques de sept écotypes de lentille de terre (Macrotyloma geocarpum (Harms) Maréchal et Baudet) au Burkina Faso. Mémoire de fin de cycle, CAP Matourkou, Bobo-Dioulasso, p. 69.

FAO. 2010. Les perspectives et les besoins alimentaires en Afrique aux échelles continentale, régionale et nationale. Rôme, Italie, p. 37.

Ghalmi N. 2011. Etude de la diversité génétique de quelques écotypes locaux de
Vigna unguiculata (L.) Walp. cultivés en Algérie. Ecole Normale Supérieure Agronomique El Harrach - Alger, Alger, p. 177.

Rémond D, Walrand S. 2017. Les graines de légumineuses: caractéristiques nutritionnelles et effets sur la santé. Innovations Agronomiques, INRA, hal01685940, p. 60.

Touré Y, Koné M, Silué, Kouadio Y J. 2013. Prospection, collecte et caractérisation agromorphologique des morphotypes de Voandzou [Vigna subterranea (L.) Verdc. (Fabaceae)] de la zone savanicole de la Côte d'Ivoire. European Scientific Journal, 9(24): 308-325. DOI: 10.19044/esj.2018.v14n15p1. 